\title{
Les affixes classificatoires des noms de nombre
}

Paul Rivet

(Apresentação de Jorge Domingues Lopes)

Rivet, Paul. 1956. Les affixes classificatoires des noms de nombre. Journal de la Société des Américanistes 45:179-187. Paris.

Há pouco mais de meio século, Rivet publicou no Journal de la Société des Américanistes o artigo "Les affixes classificatoires des noms de nombre". Nesse texto, ele demonstra como dois séculos antes o frade franciscano Pedro de la Mata descrevera com exatidão os classificadores da língua Cholón em sua Arte de la lengua cholona (1748), língua que era falada no Perú.

Antes de tecer suas considerações linguísticas, ele transcreve o conteúdo equivalente a duas páginas do texto manuscrito original da Arte, ${ }^{1}$ que trata dos "cardinales".

E, ao analisar esses dados, a primeira constatação a que Rivet chega é a de estar "diante de um sistema bastante completo de sufixos classificatórios" (grifo nosso) - ou, nas palavras de Mata, "particula distintiva para hablar con propriedad" - cuja síntese revela um conjunto de 15 afixos (alguns com formas variantes) da língua Cholón.

Como em outro artigo ele buscara aproximar essa língua de outras da família Chibcha (Cf. Rivet 1949), o pesquisador francês identifica, então, a língua Bribri, da qual ele transcreve, a título de exemplo, 8 sequências numéricas, com os respectivos sufixos destacados, e a língua Čiripó, com 4 sequências, para demonstrar que elas são tão complexas quanto à língua descrita por Mata (1748:93-94), no que se refere aos classificadores de número.

Apesar de não apresentar a mesma complexidade do sistema da língua Chólon, há outras línguas dessa família que Rivet arrola como possuidoras de algum tipo de classificador, como as línguas Move-Valiente (com 2 afixos) e Kuna (com 4 prefixos). Em seguida, completa a lista, não muito extensa, com as poucas línguas sul-americanas que apresentariam algum tipo de afixo

${ }^{1}$ Há uma cópia deste documento conservada na British Library. 
classificatório: Muchic; Aymará e Nahuatl. À parte desta lista, o autor inclui uma língua da Mesoamérica, o Maia, que possui, sem dúvida, "a mais extensa [série de sufixos classificatórios de nomes de número] entre as línguas americanas e, provavelmente, entre todas as línguas do mundo [Cf. Tozzer 1921:290-292]" (Rivet 1956:185). Rivet finaliza essa lista de línguas com classificadores citando algumas das que são faladas na América do Norte: Cœur d'Alène, Tsimshian, Haida e Ojibwa.

Conclui, então, o texto observando rapidamente que o uso de classificadores é bem mais comum em línguas melanésias, como o Florida (Nggela) e o Negone, asiáticas, como o Coreano, o Gilyak, o Aïnu, o Chinês, o Japonês, o Malaio, o Mundari e o Micronésio, e africanas, como o Zulu.

Tendo como elemento motivador deste estudo os classificadores numéricos de uma língua sul-americana, o autor inicia já um breve estudo comparativo e, mais que isso, motiva a busca por outras línguas do mundo que contenham esse recurso linguístico, cuja descrição revela o quão interessante é o estudo científico dessas línguas e, mesmo depois de tantas décadas, continua sendo uma tarefa ainda longe de ser concluída.

Por isso, a Revista Brasileira de Linguística Antropológica, neste número dedicado ao estudo de classificadores de línguas indígenas sul-americanas, republica, sob a forma de fac-símile, este artigo de Paul Rivet, prestando, assim, uma merecida homenagem ao etnólogo francês, que foi, sem dúvida, um dos pioneiros a empreender uma abordagem verdadeiramente científica no estudo linguístico-antropológico de classificadores de línguas e culturas de diferentes partes do mundo e, em particular, da América do Sul.

\section{Referências}

Mata, Pedro de la. 1748. Arte de la lengua cholona. Trujillo. Ms.

Mata, Pedro de la. 1772 [1748]. Arte de la lengua cholona. San Buenaventura del Valle [Cópia do manuscrito original por Fray Geronimo Clota].

Mata, Pedro de la. 1996 [1748]. Arte de la lengua cholona. Transcrição e edição preparada por A. Alexander-Bakkerus. Prólogo de Willem F. H. Adelaar. Madrid/ Frankfurt: Iberoamericana Vervuert. 248p. (Lingüística Misionera; 1)

Rivet, Paul. Les langues de l'ancien diocèse de Trujillo. Journal de la Société des Américanistes, t. XXXVIII, p. 1-51, 1949. Paris.

Tozzer, Alfred M. 1921. A maya grammar. Papers of the Peabody Museum of American Archaeology and Ethnology, Harvard University: with bibliography and appraisement of the works noted. Cambridge, Massachusetts, EUA: Museum of American Archaeology and Ethnology. 301p. 


\title{
LES AFFIXES CLASSIFICATOIRES DES NOMS DE NOMBRE,
}

\author{
Par Paul RIVET.
}

Dans l'article que j'ai consacré aux langues de l'ancien diocèse de 'lrujillo, où j'ai montré la parenté du Čolón-Xibito avec les langues čibča par des comparaisons lexicales, j'ai signalé l'existence de suffixes classificatoires des noms de nombre en Colón $(9,38)$.

Les faits consignés dans l'excellente grammaire manuscrite de Pedro de la MATA sont d'une grande netteté et précision $(4,93-95)$ :

Los Cardinales son los diez siguientes :

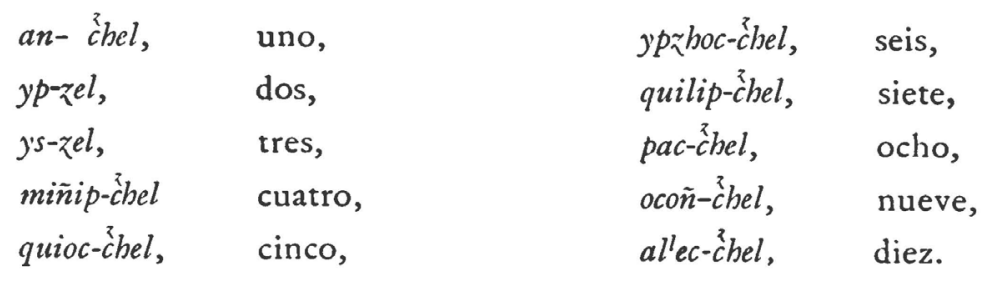

Son tan diversos los numerales en esta lengua que cada cosa que se quiera contar, para conocer lo que es, se necesita algun aditamento de particula distintiva para hablar con propriedad.

Pondré un exemplo de las cosas mas ordinarias para alguna breve noticia. El exemplo puesto sirve para cosas largas, y todo animal quadrupedo.

Para contar gente, o hombres es el siguiente :

$\begin{array}{lll}\text { an-č̉sel } & \text { (1) } & y p c^{2} h o c \\ y p-t a & (2) & q u i l i p \\ y x-t a & (3) & p a c \\ \text { miñip } & (4) & \text { ccoñ } \\ \text { quioc } & \text { (5) } & \text { alec }\end{array}$


180

SOCIÉTÉ DES AMÉRICANISTES

Para cosas redondas y todo genero de aves, frutas, etc... es el siguiente :

$\begin{array}{llll}a^{t}-c h e & \text { (I) } & y p z \text { boc-che } & (6) \\ y p-c h e & (2) & q u l i-x e & (7) \\ y x-r \hat{e} & (3) & \text { pac-che } & (8) \\ \text { miñip-che } & (4) & \text { ocoñ-che } & (9) \\ \text { quioc-che } & (5) & \text { alec-che } & \text { (10) }\end{array}$

Para ropas, vestidos, hachas, machetes, peces, etc... libros, plumas, tigeras, cuchillos, peynes, sapatos, medias:

$\begin{array}{llll}\text { au-chup } & \text { (1) } & \text { quioc-chup } & \text { (5) } \\ y p \text {-chup } & (2) & \text { ocoñ-chup } & \text { (9) } \\ y x-c b u p & (3) & \text { allec-cbup } & \text { (10) } \\ \text { miñip-chup } & \text { (1) } & & \end{array}$

Para palabras, preceptos, mandamientos, ordenanzas, etc... :

$\begin{array}{llll}\text { at-bil } & \text { (1) } & y p z o c-b i l & (6) \\ y p \text {-bil } & (2) & \text { quilix-hil } & (7) \\ y c h \text {-bil } & (3) & \text { pac-bil } & (8) \\ \text { miñip-bil } & (4) & \text { ocoñ-bil } & \text { (9) } \\ \text { quioc-bil } & \text { (5) } & \text { allec-bil } & \text { (10) }\end{array}$

Para retazos, pedazos, ñudos, junturas, etc... :

$\begin{array}{llll}a^{t}-t u s & (1) & \text { miñip-tus } & (4) \\ y p-t u s & (2) & q u i o c-t u s & (5) \\ y x-t u s & (3) & y p z o c-t u s, \text { etc... } & \text { (6) }\end{array}$

Para mitades, mendrugos, etc... :
$a^{t}$-tip
$y p-t i p$
(2)
$y x-t i p$
(3)
miñip-lip, etc... (4)

Para cosas diversas, etc..., colores, etc... :
al-liu
(I)
yx-liu, etc...
$y p-l i u$
(2)

Para vezes :

$\begin{array}{llll}a p-p o c & (1) & y^{c h} x-p o c & \text { (3) } \\ y-p o c & (2) & m i n ̃ i p-p o c, \text { etc... } & \text { (4) }\end{array}$


Para chacaras, etc... :
$a^{p}-p u c h$
$y x-p u c h$, etc...
yp-puch

Para hazes, manojos, atados, etc... :
at-chan
$y c h-c h a n$, etc...
(3)
$y p$-chan

Para vocados :
$a^{p}-p u c$
$y p-p u c$
(I)
$y c h-p u c$
(3)
(2)

Para cielos, entresuelos, quartos de casa, divisiones, doblezes de ropa, etc... :
$a^{p}$-pimoc
miñip-pimoc
(4)
yp-pimoc
$y x$-pimoc
(3)
quioc-pimoc, etc... ( 5 )

Para tropas, compañias, exercitos, manadas, etc... :
$a^{p}-p o n$
(I)
(2)
ych-pon
(3)
$y p$-pon
miñip-pon, etc...

Para pueblos, lugares, puestos, etc... :

$\begin{array}{lll}a-x u n & (\mathrm{I}) & \text { miñip-xun } \\ y p-x u n & (2) & \text { quioc-xun } \\ y x-x u n & (3) & \end{array}$

Nous nous trouvons donc devant un système très complet de suffixes classificatoires que l'on peut résumer ainsi qu'il suit :

\section{Objets à compter :}

Objets longs; quadrupèdes,

Hommes,

Objets ronds, oiseaux, fruits, etc...,

Vêtements, haches, machetes, livres, plumes, ciseaux, couteaux, peignes, souliers, bas et poissons,

\section{Suffixes :}

$-\approx e l,-$ çbel, - čbel, -ta ou rien, $-c h e,-x h,-r \hat{e}$,

$-\operatorname{chup}$ 
I 82 SOCIÉTÉ DES AMÉRICANISTES

Mots, préceptes, ordres, ordonnances, etc...,

-bil,

Fragments, morceaux, nœuds, joints, etc...,

$-t u s$,

Moitiés, morceaux de pain dur, etc...,

- tip,

Objets divers, couleurs, etc...,

-liiu,

Fois,

$-p o c$,

Champs, etc...,

-puch,

Bottes, poignées, objets ficelés, etc...,

Bouchées,

-chan,

Cieux, cntresols, chambres, divisions, plis, etc...,

-puc,

Troupes, compagnies, armées, troupeaux, etc...,

-pimoc,

Villages, lieux, postes, etc..,,

-pon,

$-x u n$.

Des affixes classificatoires de numération analogues se retrouvent dans plusieurs autres dialectes čibča.

Ce sont le Bribri et le Čiripó qui possèdent un système qui, par son développement, se rapproche le plus du système ćolón (3, I, 280-28 I, 327328).

\section{Bribri.}

Objets ronds, fruits, pierres, années :

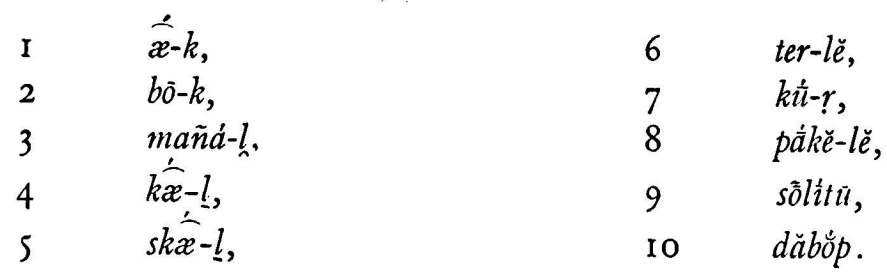

Maisons, foyers, tombeaux familiaux :

\begin{tabular}{|c|c|c|c|}
\hline I & 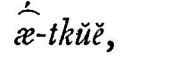 & 5 & $s k x-l \breve{u}$, \\
\hline 2 & 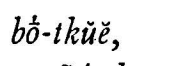 & 6 & tæædŭ-lŭ̌ \\
\hline 3 & 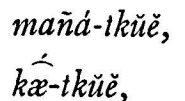 & IO & 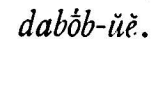 \\
\hline
\end{tabular}

Arbres, fleuves, bétail, jaguars, poissons, cordes, flèches, arcs, sarbacanes :

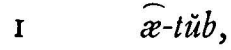

$2 b \dot{o}-t u ̈ b$,

Hommes, personnes, dieux :

I $\dot{\mathfrak{x}}-k \dot{u}-l$,

$2 b \bar{o}-t$
Io dabópp-lüb.

$\begin{array}{ll}3 & \operatorname{maña}-t \\ 4 & \dot{k}-l\end{array}$


Corbeilles, chemises, pantalons, dents, mains, oiseaux, mois, temps, jours, champs de maïs, objets :

$\begin{array}{cccc}\dot{\hat{a}}-t, & 3 & \operatorname{ma} \tilde{n} \dot{a}-t, \\ 2 & b \bar{o}-t, & 4 & k \dot{a}-l .\end{array}$

Arbres de cacao, et autres arbres fruitiers :

\begin{tabular}{|c|c|c|c|}
\hline I & $\stackrel{\dot{x} r-k a t}{,}$ & 5 & skêx-r-kat, \\
\hline 2 & $b o ̛ ́-r-k a t$, & 6 & $\stackrel{\dot{x}}{t \mathfrak{x}-r-k a t}$ \\
\hline 3 & mañáa-r-kat, & 7 & $-k a t$ \\
\hline & $k \widetilde{x}-r-k a t$ & & \\
\hline
\end{tabular}

Fardeaux :

\begin{tabular}{|c|c|c|c|}
\hline I & $\dot{\bar{x}-} d_{2}^{\star} \breve{u} k$ & 4 & 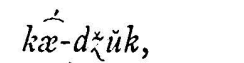 \\
\hline & $b \dot{\bar{o}}-d_{\varkappa} \breve{\imath} k$, & 5 & $s \hat{k a-d z ̌ u k, ~}$ \\
\hline & 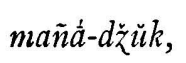 & 6 & 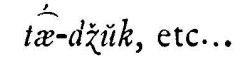 \\
\hline
\end{tabular}

Jours :

demain,

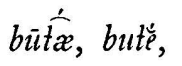

dans 9 jours,

sünîto-ki,

après-demain,

$b \bar{o}-i k \hat{i}$,

dans 3 jours,

măña-èk,

dans ro jours,

$d \bar{a} b o ̈ ́ p-k i$,

dans 4 jours,

kíx-k,

hier,

čki,

dans 5 jours,

skæ̀-k,

avant-hier,

bò-kri,

il $\mathrm{y}$ a 3 jours,

mañá-l-iki,

dans 6 jours,

tar-iki,

il y a 4 jours,

k̇̀-l-iki,

dans 7 jours,

$k i$-iki,

il y a 5 jours,

sìm-n-iki,

dans 8 jours,

$p \dot{a}-i k i$,

il $y$ a 6 jours,

d) $\dot{x}-r-i k i$.

\section{Ciripó.}

Bananes :

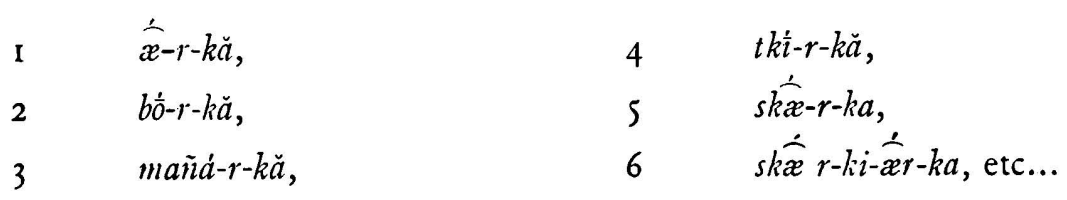

Années :

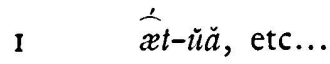


Poissons :

\begin{tabular}{|c|c|c|c|}
\hline I & $\dot{x}-t-a ̈ b \breve{a}$ & 4 & $t k \hat{\imath}-t-a \breve{b} b \ddot{e}$, \\
\hline & $b \dot{o}-t-o b e$ & 5 & $t-\breve{a} b \grave{e}$ \\
\hline
\end{tabular}

Orangers et autres arbres fruitiers :

\begin{tabular}{|c|c|c|}
\hline & $\dot{x}-k a ̆ b a ̆$, & 4 \\
\hline & $\begin{array}{l}\text { bó }-r o, \\
\text { manắ-t, }\end{array}$ & $S$ \\
\hline
\end{tabular}

En Move-Valiente (groupe guaymi), les faits sont moins complexes (7).

En face de la numération normale, il y a une numération spéciale pour les jours, avec le suffixe -era et pour la monnaie, avec le préfixe mana- :

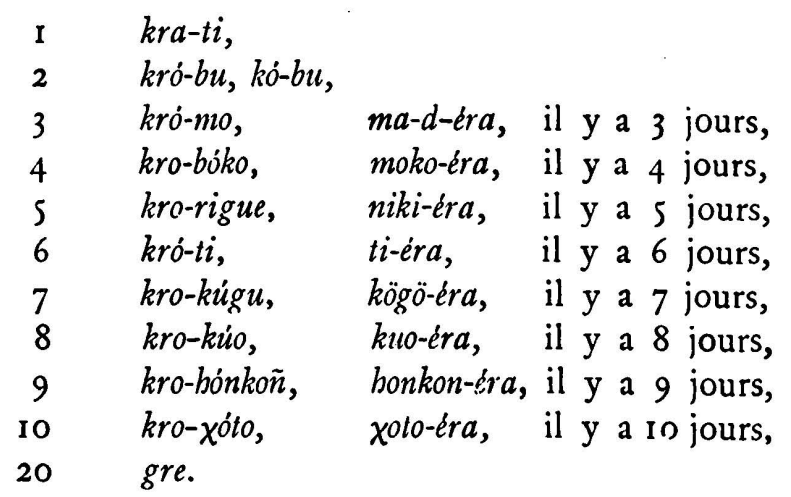

$\begin{array}{lllr}\text { maná-ni, } & \text { I real, } & \text { mana-kögu, } & 7 \text { reales, } \\ \text { maná-mu, } & 2 \text { reales, } & \text { mana-kúo, } & 8 \text { reales, } \\ \text { mana-mo, } & 3 \text { reales, } & \text { maná-bókon, } & 9 \text { reales, } \\ \text { maná-bóko, } & 4 \text { reales, } & \text { maná- } x \dot{o} t o, & \text { ro reales, } \\ \text { mana-dikie } & 5 \text { reales, } & \text { maná-gre, } & 20 \text { reales. } \\ \text { maná- } n-t i, & 6 \text { reales, } & & \end{array}$

En Kuna, il y a quatre préfixes classificatoires (2, IOI-IO2).

Noms variés (animaux inclus),

kua-,

Crayons, cigarettes, fusils, poissons, $\quad k a-$,

Personnes et animaux, war-,

Arbres et plantes, sar-. 
D'autres affixes ne sont que des noms communs :

kia-, pièce, utilisé pour dénombrer des ficelles, or-, dérivé de olo, morceau, employé pour dénombrer des objets ronds, matta-, feuille, lorsqu'il s'agit de papier.

En dehors de la famille čibča, les affixes classificatoires des noms de nombre sont rares en Amérique.

En Mučik (6, 68-69, I29-I 30), on a pour compter le temps au-dessus de ro : nok ssop käss, $\quad 40$ jours, et non pas: nok pong käss, pak ssop für, 20 ans, " pak pong für,

pour compter des fruits, et particulièrement des épis de maïs :

na chokij mang,
pak chokij mang, épis de mais,
20 épis de maïs,

pour compter certains fruits :

pak chiäng ćbun, 200 courges, et non pas: pak paläk ćbun, ssok chiäng $\bar{o} p, \quad 300$ patates douces, $\quad$ sok paläk oo $p$, nok chiäng äp, 400 gousses de piment, „ nok paläk äp.

pong signifie "pierre", ssop "corde"; nous ignorons le sens de chokij, de chiäng et de paläk.

Si l'on compte par paire, on emploie:

$l u k$, pour les fruits, légumes, œufs, objets ronds :

na-luk, une paire,

pak-luk, deux paires,

sck-luk, trois paires,

feläp, pour les volailles, les vases et les objets plus volumineux :

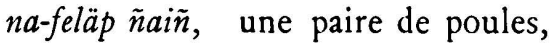

pak-feläp fellu, deux paires de canards.

L'Aymará ne présente qu'un seul exemple de l'emploi d'un suffixe classificatoire des noms de nombre:

$m a i-n i$, un, et $p a-n i$, deux, servent pour compter des êtres humains, ma$y a$ et $p a-y a$ pour compter des animaux ou des objets inanimés $(5,68)$.

N. M. Holmer signale des traces de ce système en Nahuatl (2, I02).

Le Maya possède une série de 80 suffixes classificatoires des noms de nombre qui est sans aucun doute la plus étendue parmi les langues américaines et probablement parmi toutes les langues du monde (11, 290-292). 
Signalons encore l'existence de ce système en Cour d'Alène (groupe salish) $(\mathbf{8}, 645)$, en Tsimšian, en Haida, en Odžibue $(\mathbf{1 0}, \mathbf{2 3} 8)$.

Il n'est pas dans mon propos de signaler les langues extra-américaines où l'on rencontre la même particularité. Je rappellerai seulement que le procédé est fréquent dans les langues mélanésiennes (1, 24I-242).

Dans certaines de ces langues, le suffixe classificatoire pour les dizaines suffit à lui seul pour déterminer l'objet dont il s'agit, sans que le nom de cet objet soit exprimé.

C'est ainsi qu'à Florida :

na-kua, signifie dix œufs (lolu, œuf)

na-banara, dix corbeilles de nourriture

$n a-g o b i\left\{\begin{array}{l}\text { dix canots } \\ \text { dix puddings }\end{array} \quad\right.$ (vaka, canot)

$n a-p a n g a \begin{cases}\text { dix porcs } & (\text { bolo, porc) } \\ \text { dix oiseaux } & (\text { manu, oiseau) } \\ \text { dix poissons } & (\text { iga }, \text { poisson) } \\ \text { dix opossums } & \end{cases}$

et à Fiji : a-ıduudu signifie dix canots (waga, canot)

$$
\begin{aligned}
& \text { a-rara, } \\
& \text { a-soga, } \quad \text { dix porcs (vuaka, porc) } \\
& \text { a-bola, } \quad-\text { dix volailles (toa, volaille) } \\
& \text { dixsons (ika, poisson). }
\end{aligned}
$$

Il existe beaucoup de suffixes semblables pour désigner des dizaines de noix de coco, de fruits de l'arbre à pain, de crabes, de coquillages, de régimes de bananes, de corbeilles de noix.

A Nengone, on joint le mot naiu, frapper, au non de nombre qui désigne des lances, deder, voler, au nom de nombre qui désigne des oiseaux en vol ou $t e$, être assis, au nom de nombre qui désigne des oiseaux posés.

Des faits analogues se retrouvent en Coréen, en Gilyak, en Ainu, en Chinois, en Japonais, en Malais, en Munda, en Micronésien, dans quelques langues de Nouvelle-Guinée (10, 238, 250, 775).

Enfin, dans le domaine africain, la numération participe au système classificatoire en Zulu (langue bantu), suivant un renseignement qu'a bien voulu me communiquer E. Benveniste.

De cette rapide revue, il ressort que l'usage des affixes classificatoires des noms de nombre semble se retrouver surtout dans les langues péripacifiques, mais il est évident que l'enquête aurait besoin d'être poussée plus avant pour confirmer cette conclusion. De toutes façons, l'exemple du Colón-Xibito, par sa complexité, est comparable à celui du Maya. 


\section{BIBLIOGRAPHIE.}

1. Codrington (R. H.). - The Melanesian langutges. Oxford, 1885.

2. Holmer (Nils M.). - Critical and comparatize grammar of the Cuna language. Etnologiska Studier, 14. Göteborg, 1947, [12] + 219 p.

3. Lehmaxn (Walther). - Kentral-Amerika. I. Teil. Die Sprachen Lentral-Amerikas. Berlin, 2 vol., I920.

4. Mata (Pedro de la). - Arte de la lengua cholona. Trujillo, 1748. Ms. de la Bibliothèque du British Museum. P. 5345, Add. 25322.

5. Mrddendorf (E. W.). - Die Aimará-Sprache. Die einheimischen Sprachen Perus, t. V. Leipzig, 1891.

6. - Das Mucbik oder die Chimu-Sprache. Die einheimischen Sprachen Perus, t. VI. Leipzig, 1892.

7. Pinart (Alfonso L.). - Vocabulario caslellano-guaymie. Dialcctos Move-Valiente, Norteño y Guaymie-Penonomeño. Paris, I 892.

8. Reichard (Gladys A.). - Cour d'Alene. Handbook of american indian languages. New York, Columbia University Press, Part 3, 1933-1938, p. 517-707.

9. River (Paul). - Les langues de l'ancien diocise de Trujillo. Journal de la Société des Américanistes. Paris, nouvelle série, t. XXXVIII, 1949, p. I-51.

10. Royen (Gerlach). - Die nominalen Klassifikations-Systeme in den Sprachen der Erde. Bibliotheqque linguistique - Anthropos -, t. IV. St. Gabriel-Mödling, bei Wien, 1929.

11. Tozzer (Alfred M.). - A maya grammar. Papers of the Peabody Museum of american archaeology and ethnology, Harvard Uuiversity. Cambridge, t. IX, I92I. 